\title{
ИССЛЕДОВАНИЕ ВЛИЯНИЯ КОНТАКТНОГО ВЗАИМОДЕЙСТВИЯ ЗАГОТОВКИ И ИНСТРУМЕНТА НА ДИНАМИЧЕСКИЕ ХАРАКТЕРИСТИКИ ТОКАРНОГО СТАНКА
}

\author{
Danylchenko M., Petryshyn A. \\ National Technical University of Ukraine «Igor Sikorsky Kyiv Polytechnic Institute», Kyiv, Ukraine

\section{STUDY OF WORKPIECE AND TOOL CONTACT INTERACTION INFLUENCE ON DYNAMIC CHARACTERISTICS OF A LATHE}

\begin{abstract}
Разработана динамическая модель упругой системы токарного станка с учетом контактного взаимодействия заготовки и инструмента. Получены аналитические зависимости для расчета частот собственных колебаний упругой системы станка при учете процесса резания в виде дополнительной упругой связи в месте контакта заготовки и инструмента. Представлена методика расчета жесткости этой связи с учетом упругих смещений заготовки и инструмента. На токарно-винторезном станке мод. 16Б05П экспериментально определены частоты собственных колебаний шпинделя с закрепленной в патроне заготовкой без ее поджатия резцом и при пяти вариантах поджатия на разных вылетах. Для этого же станка и таких же условий взаимодействия заготовки с резиом проведено численное моделирование частот собственных колебаний упругой системы станка. Результаты моделирования соответствуют экспериментальным значениям частот собственных колебаний и достаточно точно отражают тенденции их изменения при изменении массоинериионных характеристик заготовки и условий ее взаимодействия с резиом. Это подтверждает целесообразность учета процесса резания в замкнутых динамических моделях металлорежущих станков в виде дополнительной упругой связи в месте контакта заготовки и инструмента
\end{abstract}

Ключевые слова: токарный станок; шпиндельный узел; динамическая модель; частоты собственных колебаний; эксперимент; численное моделирование.

\section{Введение}

Точность обработки на металлорежущих станках в значительной степени зависит от их динамических свойств и условий обработки, которые могут сопровождаться возникновением интенсивных вибраций. Успешность борьбы с ним, прежде всего, зависит от понимания физических причин их возникновения. Наиболее эффективным методом выявления этих причин является исследование поведения механической системы станка на соответствующих динамических моделях. Поэтому вопрос адекватности учета в динамических моделях реально действующих процессов является актуальным.

В динамических моделях металлорежущих станков механическая система, как правило, представляется в виде упругой системы (УС), состоящей из подсистем инструмента и заготовки, нагруженных силой резания. Взаимодействие инструмента и заготовки происходит через процесс резания, что учитывается в модели обратной связью между этим процессом и УС [1]. Таким образом, процесс резания заменяется внешней силой, действующей на инструмент и заготовку без учета фактического контактного взаимодействия между ними. То есть механически замкнутая система станка представляется в виде двух механически разомкнутых подсистем инструмента и заготовки. В итоге, при исследовании динамических свойств УС станка с использование этой модели можно определить только собственные частоты отдельных элементов УС, а не замкнутой системы целиком.

Вместе с тем, в [2] отмечается, что действие нормальной составляющей силы резания в плоскости среза подобно действию сил упругого взаимодействия контактирующих твердых тел (инструмента и заготовки). Это позволяет отнести эту составляющую силы резания к «упругой» и ввести понятие «жесткости резания». Величина этой жесткости $k_{p}$ определяется отношением нормальной составляющей силы резания к изменению толщины среза и изменяется в соответствии с законами резания [2].

Введение понятия «жесткости резания» позволяет в расчетной схеме УС станка учитывать процесс резания дополнительной упругой связью в месте «соединения» инструмента и заготовки и таким образом учитывать фактическую замкнутость УС при расчете ее динамических характеристик. 
Именно такой подход используется при исследовании динамического воздействия подвижной нагрузки на точность обработки маложестких деталей $[3,4]$. Динамические модели процессов обработки здесь представлены в виде эквивалентной трехмассовой системы «инструментальная головка - заготовка шпиндельный узел» [3] и в виде закрепленной в упругих опорах заготовки с распределенной массой [4]. Обе модели предусматривают преобладающее влияние упругости заготовки на динамику процесса обработки. Влияние механической системы станка учитывается в упрощенном виде.

Более общая динамическая модель станка, учитывающая не только влияние заготовки, но и влияние формообразующих узлов станка представлена в [5]. В этой модели учтены жесткости закрепления заготовки (инструмента) в шпинделе, установки шпинделя в корпус, а корпуса на основу станка, что предусмотрено рекомендациями по разработке динамических моделей шпиндельных узлов металлорежущих станков [6]. Расчетная часть модели [5] основана на декомпозиции замкнутой упругой системы станка с использованием метода динамических податливостей и применении метода переходных матриц для описания упругих свойств элементов подсистем [7].

Модель [5] предлагается использовать как базовую для разработки динамической модели токарного станка при продольном точении. Это позволит с большей степенью достоверности оценить характер и степень влияния контактного взаимодействия заготовки и инструмента на динамические характеристики (частоты собственных колебаний) УС станка.

Цель.

Целью этой работы является численное и экспериментальное исследование влияния контактного взаимодействия заготовки и инструмента на динамические характеристики (частоты собственных колебаний) УС токарного станка.

Динамическая модель упругой системы токарного станка при продольном точении.

Исследование влияния контактного взаимодействия заготовки и инструмента на частоты собственных колебаний УС токарного станка проводится на станке с «разгруженным приводом», размещенным в межопорной части шпинделя. При такой конструкции привода вес приводного шкива не воспринимается телом шпинделя, поэтому в динамической модели влияние привода учитываться не будет.

Динамическая модель УС токарного станка при продольном точении представлена в виде замкнутой механической колебательной системы, состоящей из трех парциальных подсистем: заготовки (подсистема 1, индекс $s=1$ ), шпинделя с патроном (подсистема 2, индекс $s=2$ ) и суппорта с инструментом (подсистема 3 , индекс $s=3$ ). Соответствующие конструктивная и расчетная схемы представлены на рис. 1.

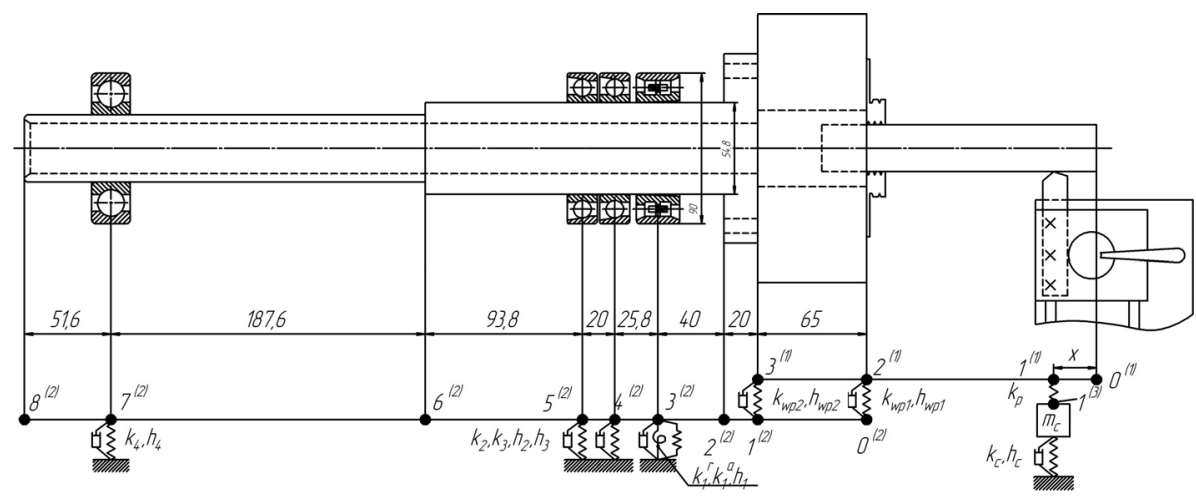

Рис. 1. Конструктивная и расчетная схемы замкнутой механической колебательной системы токарного станка при продольном точении

Первая подсистема ( $s=1$ ) рассматривается в виде стержня с распределенной массой, состоящего из трех участков $(u=3)$. В сечении $1^{(1)}$ заготовка контактирует с инструментом, что учитывается упругой связью с жесткостью $k_{p}$. При размыкании системы эта связь учитывается в подсистеме заготовки. В сечениях $2^{(1)}$ и $3^{(1)}$ заготовка соединяется со шпинделем (сечения $0^{(2)}$ и $1^{(2)}$ шпинделя), что учитывается упругими и демпфирующими связями с жесткостями $k_{w p 1}, k_{w p 2}$ и коэффициентами демпфирования $h_{w p 1}, h_{w p 2}$ соответственно.

Вторая подсистема ( $s=2$ ) рассматривается в виде упруго закрепленного на станине станка стержня с распределенной массой. Стержень состоит из восьми участков $(u=8)$. В сечениях $3^{(2)}, 4^{(2)}, 5^{(2)}$ и $7^{(2)}$ стержня размещены подшипники опор, что учитывается упругими и демпфирующими связями шпинделя со станиной станка с жесткостями $k_{1}^{r}, k_{1}^{a}, k_{2}, k_{3}, k_{4}$ и коэффициентами демпфирования $h_{1}, h_{2}, h_{3}, h_{4}$ соответственно. 
Третья подсистема $(s=3)$ рассматривается в виде сосредоточенной массы $m_{c}$, упруго закрепленной на станине станка, что учитывается жесткостью $k_{c}$ и коэффициентом демпфирования $h_{c}$ соответственно.

Декомпозицию замкнутой механической колебательной системы, представленной на рис. 1 , проведем с использованием метода динамических податливостей $[5,8]$. Согласно этому методу сложные механические системы разделяются на более простые парциальные подсистемы путем устранения связей между ними. В направлении отброшенных связей прикладываются их реакции, которые затем определяют из условия совместности деформаций подсистем. Схема разделения системы (рис. 1) представлена на рис. 2.

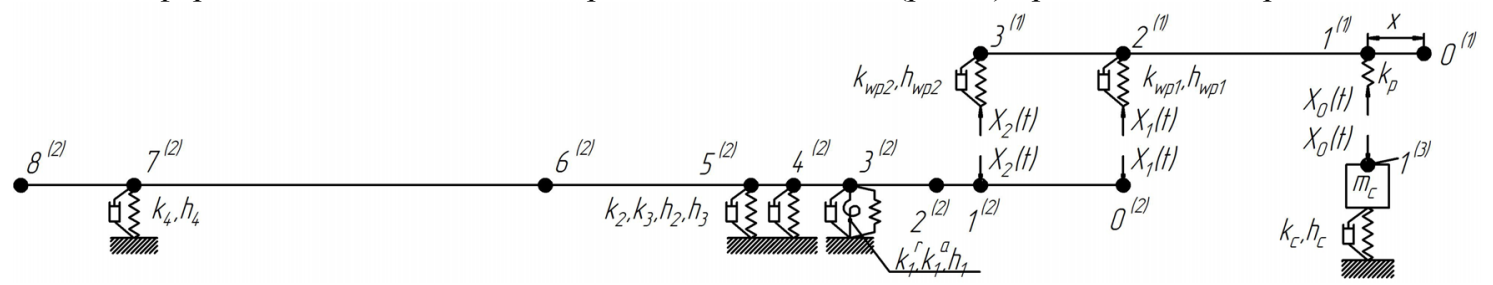

Рис. 2. Схема разделения замкнутой механической колебательной системы токарного станка при продольном точении

Условия совместности деформаций подсистем представляются в виде условий равенства амплитуд линейных перемещений в точках отделения подсистем:

- для подсистем 1 и 2:

$$
\left\{\begin{array}{l}
\alpha_{21}^{(1)} \cdot X_{0}+\left(\alpha_{22}^{(1)}+\frac{1}{k_{w p 1}}\right) \cdot X_{1}+\alpha_{23}^{(1)} \cdot X_{2}=-\alpha_{00}^{(2)} \cdot X_{1}-\alpha_{01}^{(2)} \cdot X_{2} \\
\alpha_{31}^{(1)} \cdot X_{0}+\alpha_{32}^{(1)} \cdot X_{1}+\left(\alpha_{33}^{(1)}+\frac{1}{k_{w p 2}}\right) \cdot X_{2}=-\alpha_{10}^{(2)} \cdot X_{1}-\alpha_{11}^{(2)} \cdot X_{2}
\end{array},\right.
$$

- для подсистем 1 и 3 :

$$
\left(\alpha_{11}^{(1)}+\frac{1}{k_{p}}\right) \cdot X_{0}+\alpha_{12}^{(1)} \cdot X_{1}+\alpha_{13}^{(1)} \cdot X_{2}=-\alpha_{c}^{(3)} \cdot X_{0},
$$

где $X_{j}$ - амплитуды реакций отброшенных связей, $j=0,1,2 ; \alpha_{i j}^{(s)}$ - гармонические коэффициенты влияния подсистем $s$, а именно амплитуды перемещения в $i$-ой точке от единичной гармонической силы, приложенной в $j$-ой точке; $\alpha_{c}^{(3)}$ - локальная динамическая податливость третьей подсистемы: $\alpha_{c}^{(3)}=\frac{1}{k_{c}-m_{c} \cdot \omega^{2}},[8]$.

После преобразования (1) и (2), общая система уравнений совместности деформаций в точках размыкания подсистем будет иметь вид:

$$
\left\{\begin{array}{l}
\left.\left(\alpha_{11}^{(1)}+\frac{1}{k_{p}}\right)+\alpha_{c}^{(3)}\right) X_{0}+\alpha_{12}^{(1)} \cdot X_{1}+\alpha_{13}^{(1)} \cdot X_{2}=0 \\
\alpha_{21}^{(1)} \cdot X_{0}+\left(\left(\alpha_{22}^{(1)}+\frac{1}{k_{w p 1}}\right)+\alpha_{00}^{(2)}\right) \cdot X_{1}+\left(\alpha_{23}^{(1)}+\alpha_{01}^{(2)}\right) \cdot X_{2}=0, \quad \text { или }[\mathbf{D}(\omega)] \cdot[\mathbf{X}]=0, \\
\alpha_{31}^{(1)} \cdot X_{0}+\left(\alpha_{32}^{(1)}+\alpha_{10}^{(2)}\right) \cdot X_{1}+\left(\left(\alpha_{33}^{(1)}+\frac{1}{k_{w p 2}}\right)+\alpha_{11}^{(2)}\right) \cdot X_{2}=0
\end{array}\right.
$$

где $[\mathbf{X}]$ - вектор амплитуд гармонических реакций отброшенных связей, $[\mathbf{X}]=\left(X_{0}, X_{1}, X_{2}\right)^{T} ;[\mathbf{D}(\omega)]-$ матрица гармонических коэффициентов влияния:

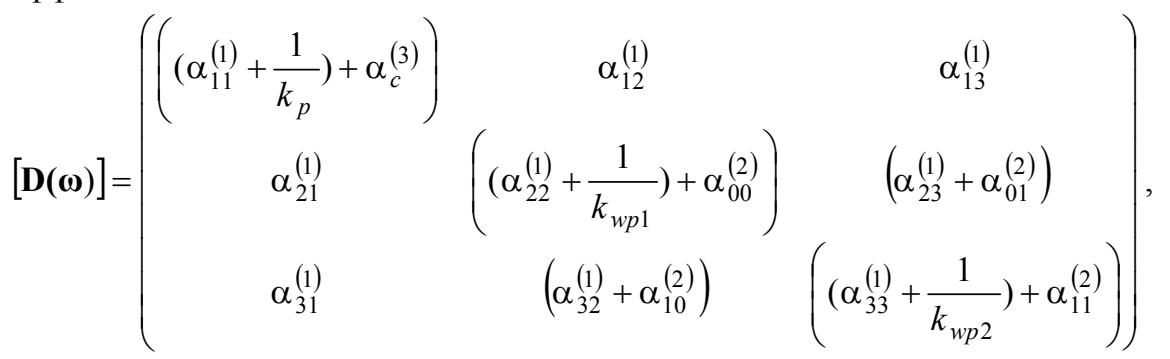

Из системы уравнений (3) определяются реакции отброшенных связей, а потом амплитуды $q_{i}^{(s)}$ $(i=0 \div u)$ поперечных (радиальных) перемещений характерных точек парциальных подсистем:

- подсистема 1 
- подсистема 2

$$
q_{i}^{(1)}=\alpha_{i 1}^{(1)} \cdot X_{0}+\alpha_{i 2}^{(1)} \cdot X_{1}+\alpha_{i 3}^{(1)} \cdot X_{2}
$$

- подсистема 3

$$
q_{i}^{(2)}=-\alpha_{i 0}^{(2)} \cdot X_{1}-\alpha_{i 1}^{(2)} \cdot X_{2}
$$

$$
q_{c}^{(3)}=-\alpha_{c}^{(3)} \cdot X_{0},
$$

Собственные частоты системы определяются из условия $\operatorname{det}[\mathbf{D}(\omega)]=0$.

Расчет гармонических коэффициентов влияния $\alpha_{i j}^{(s)}$ подсистем проводился по методике [7] с использованием метода начальных параметров в матричной формулировке (метода переходных матриц) [9].

В общем случае «жесткость резания» $k_{p}$ в (3) и (4) представляется в виде коэффициента связи между нормальной составляющей силы резания $P_{r}$ и фактической величиной глубины резания $H_{r e a l}$.

С учетом общеизвестной зависимости для определения составляющей силы резания $P_{r}$ при точении: $P_{r}=C_{p} H_{\text {real }}^{x} S^{y} V^{n} k$, значение $k_{p}$ определяется зависимостью [10]:

$$
k_{p}=\partial P_{r} / \partial H_{\text {real }}=C_{p} x H_{\text {real }}^{x-1} S^{y} V^{n} k .
$$

Фактическая величина глубины резания $H_{\text {real }}$ определяется относительным смещением заготовки и инструмента:

$$
H_{\text {real }}=H_{p r}-q_{c m}-q_{\partial u н}
$$

где $H_{p r}$ - заданная глубина резания; $q_{c m}$ и $q_{\text {дин }}$ - постоянная и переменная составляющие относительного смещения заготовки и инструмента.

Постоянная составляющая определяется по результатам статического расчета, а переменная составляющая - по результатам динамического расчета с использованием зависимостей (5) и (7):

$$
q_{\text {дин }}=q_{1}^{(1)}-q_{c}^{(3)}=\left(\alpha_{11}^{(1)}-\alpha_{c}^{(3)}\right) \cdot X_{0}+\alpha_{12}^{(1)} \cdot X_{1}+\alpha_{13}^{(1)} \cdot X_{2} .
$$

Исследование влияния контактного взаимодействия заготовки и инструмента на динамические характеристики (частоты собственных колебаний) упругой системы токарного станка.

Исследование проводилось на токарно-винторезном станке повышенной точности мод. 16Б05П, оснащенном трехкулачковым патроном СТ160В-Ф4. В качестве заготовок использовались стальные цилиндрические заготовки длиной 174 мм: сплошная - с внешним диаметром 28 мм, и полая - с внешним диаметром 28 мм и толщиною стенки 3 мм. Длина консольной части (вылет) заготовок при зажиме в патроне составляет 122 мм.

Суть исследования состояла в численном и экспериментальном определении частот собственных колебаний зажатой в трехкулачковом патроне заготовки при ее поджиме резцом на разных вылетах (без резания).

Для этого случая «жесткость резания» $k_{p}$ в расчетной модели в (3) и (4) определяется по зависимости (8) из условия $H_{\text {real }}=H_{p r}$ для продольного точения заготовки из стали 45 резцом с материалом режущей части Т15К8. Режимы резания: глубина резания $H_{p r}=0,5$ мм, осевая подача $s=0,4$ мм/об, скорость резания $V=160$ м/ мин. Радиальная составляющая силы резания для этих условий $P_{r}=245 H$, а «жесткость резания» $k_{p}=0,404 H /$ мкм.

Кроме этого в расчетной модели принято:

- радиальная и угловая жесткость двухрядного роликового подшипника мод. 4-318211 передней опоры шпинделя $k_{1}^{r}=502 \mathrm{H} /$ мкм и $k_{1}^{a}=1205 \mathrm{H} \cdot$ мкм $/$ рад;

- радиальная жесткость радиально-упорных шариковых подшипников мод. 4-46111Л передней опоры шпинделя $k_{2}=k_{3}=257 \mathrm{H} /$ мкм;

- жесткость радиального шарикового подшипника мод. 4-308 задней опоры шпинделя $k_{4}=365 H /$ мкм ;

- коэффициенты демпфирования подшипников шпинделя $h_{1}=h_{2}=h_{3}=h_{4}=2 \mathrm{H} \cdot \mathrm{c} / \mathrm{Mm}$

- жесткость и коэффициенты демпфирования стыка патрона с заготовкой $k_{w p 1}=k_{w p 2}=12 \mathrm{H} /$ мкм и $h_{w p 1}=h_{w p 2}=0.3 \mathrm{H} \cdot \mathrm{c} / \mathrm{MM}$;

- масса и жесткость закрепления суппорта $m_{c}=50 \kappa 2, k_{c}=70 \mathrm{H} /$ мкм .

В ходе эксперимента проводилось измерение свободных затухающих колебаний зажатой в трехкулачковом патроне заготовки при импульсном нагружении (путем направленного удара). Схема измерения приведена на рис. 3 . 
Измерение проводилось с использованием закрепленного на заготовке 1 пьезоэлектрического датчика 2 мод. РСВ 353В15. Аналоговый сигнал с датчика 2 усиливался предусилителем 3 мод. РСВ 480 Е09 и поступал в аналого-цифровой преобразователь 4 мод. NI USB-9215. Полученный с помощью АЦП 4 цифровой сигнал сохранялся и обрабатывался на ПК 5 с помощью программной среды Matlab.

В результате измерений и последующих преобразований были получены спектры свободных колебаний сплошной и полой заготовок без поджатия резцом (рис. 4), и аналогичные спектры для пяти вариантов поджатия резцом на вылетах 122, 102, 82, 62, i 42 мм соответственно (рис. 5).
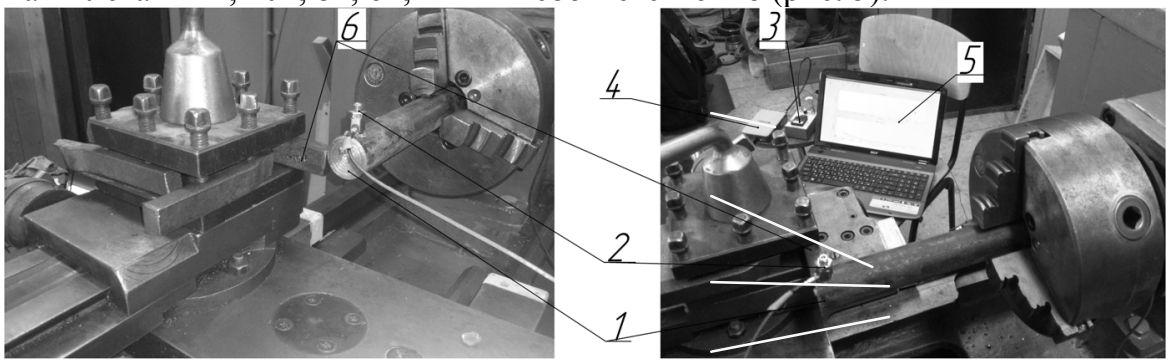

Рис. 3. Схема измерения колебаний заготовки: 1 - заготовка; 2 - пьезоэлектрический датчик;

3 - предусилитель; 4 - аналогово-цифровой преобразователь; 5 - персональный компьютер; 6 - резец

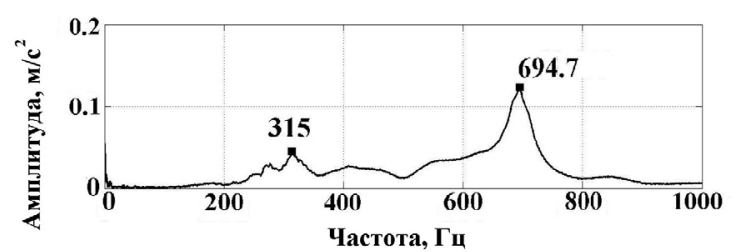

a

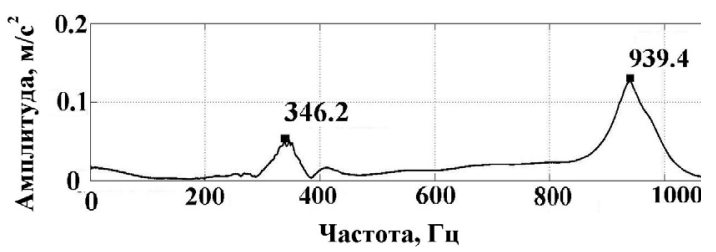

б

Рис. 4. Спектры колебаний заготовки без поджатия резцом: а) сплошной; б) полой
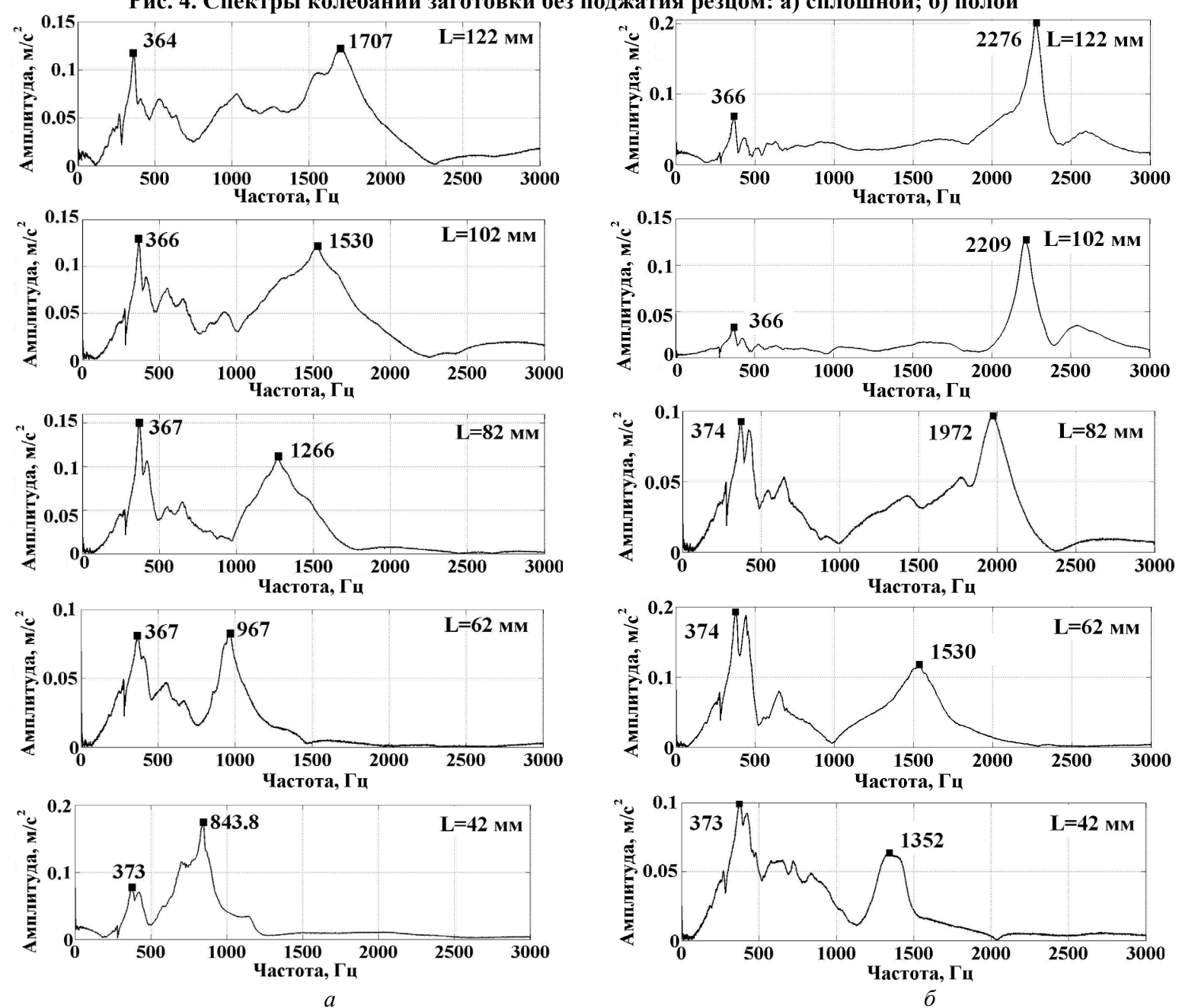

Рис. 5. Спектры колебаний заготовки при поджатии резцом на расстоянии $L$ от патрона для заготовки: а) сплошной; б) полой 
Как свидетельствуют результаты эксперимента при исследовании колебаний зажатой в патроне заготовки без ее поджатия резцом (рис.4), в частотном диапазоне от 0 до 3000 Ги наблюдаются две зоны колебаний с повышенными амплитудами: в диапазоне 315 - 346 Ги и 695 - 939 Ги. Как показали результаты численного моделирования, первая зона соответствует колебаниям на собственной частоте шпинделя $\left(p_{I}\right)$, а вторая - на собственной частоте заготовки $\left(p_{2}\right)$. При этом необходимо отметить, что значения частот собственных колебаний разные для полой и сплошной заготовок. Так, первая собственная частота системы $p_{1}$, изменяется незначительно (346 Ги для полой заготовки и 315 Гu - для сплошной), что объясняется незначительным вкладом заготовки в изменение массо-инерционных характеристик системы «шпиндель-заготовка». Вторая же собственная частота системы $p_{2}$, изменяется значительно $(939,4$ Ги для полой заготовки и 694,7 Гu - для сплошной), что объясняется существенным (по сравнению с изменением жесткости) изменением массоинерционных характеристик самой заготовки. Кроме этого надо указать, что значения собственных частот не изменялись ни при изменении места крепления датчика на заготовке, ни при изменении места приложения импульсного возбуждения.

При исследовании колебаний зажатой в патроне заготовки с ее поджатием резцом (рис. 5) видно, что первая собственная частота системы $p_{1}$ изменяется незначительно: в диапазоне 366-373 Гц для полой заготовки и 364-373 Гц - для сплошной. Как видно, значения $p_{1}$ выше соответствующих значений, полученных в результате измерений колебаний без поджатия заготовки резцом. Это объясняется увеличением жесткости системы. То есть резец в замкнутой динамической системе выступает в качестве дополнительной опоры. А увеличение $p_{1}$ по мере приближения точки поджатия к торцу патрона связано с тем, что эта опора является подвижной.

Вторая собственная частота системы $p_{2}$ (рис. 5) изменяется намного значительней. Значение этой частоты уменьшается по мере приближения точки поджатия к торцу патрона: для полой заготовки - с 2276 до 1352 Гц, и для сплошной - с 1707 до 843,8 Гц. Это также связано с подвижностью дополнительной опоры.

Сравнительные результаты численного и экспериментального исследования сведены в таблицу 1.

Таблица 1

Экспериментальные/расчетные значения собственных частот системы

\begin{tabular}{|c|c|c|c|c|}
\hline \multirow{2}{*}{$\begin{array}{c}\text { Расстояние от резца до } \\
\text { торца патрона }\end{array}$} & \multicolumn{2}{|c|}{ Полая заготовка } & \multicolumn{2}{|c|}{ Сплошная заготовка } \\
\hline & $p_{l}, \Gamma u$ & $p_{2}, \Gamma u$ & $p_{l}, \Gamma u$ & $p_{2}, \Gamma u$ \\
\hline \multicolumn{5}{|c|}{ Без поджатия заготовки резцом } \\
\hline & $346 / 331$ & $939 / 937$ & $315 / 318$ & $695 / 721$ \\
\hline \multicolumn{5}{|c|}{ С поджатием заготовки резцом } \\
\hline $\mathrm{L}=42 \mathrm{MM}$ & $373 / 369$ & $1352 / 1368$ & $373 / 368$ & $844 / 851$ \\
\hline $\mathrm{L}=62 \mathrm{MM}$ & $374 / 367$ & $1530 / 1640$ & $367 / 367$ & $967 / 973$ \\
\hline $\mathrm{L}=82 \mathrm{MM}$ & $374 / 366$ & $1972 / 1920$ & $367 / 366$ & $1266 / 1275$ \\
\hline $\mathrm{L}=102 \mathrm{MM}$ & $366 / 365$ & $2209 / 2158$ & $366 / 364$ & $1530 / 1552$ \\
\hline $\mathrm{L}=122 \mathrm{MM}$ & $366 / 363$ & $2276 / 2265$ & $364 / 362$ & $1707 / 1698$ \\
\hline
\end{tabular}

Как свидетельствуют данные табл. 1, результаты численного моделирования частот собственных колебаний правильно отражают тенденции их изменения в зависимости от расстояния между резцом и торцом патрона. Это подтверждает адекватность разработанной динамической модели токарного станка и целесообразность учета процесса резания в замкнутых динамических моделях металлорежущих станков в виде дополнительной упругой связи в месте контакта заготовки и инструмента.

\section{Выводы}

В общем, результаты исследования свидетельствуют о том, что динамические характеристики (собственные частоты) токарного станка существенно зависят от условий контактного взаимодействия заготовки с инструментом и изменяются в широком диапазоне в высокочастотной области. Изменение значений собственных частот в таком диапазоне может привести к возникновению интенсивных вибраций и ухудшению качества обрабатываемых поверхностей. В динамических моделях токарных станков, для учета контактного взаимодействия заготовки с инструментом целесообразно в месте их контакта вводить в расчетную схему дополнительную подвижную упругую опору, жесткость которой определяется фактической величиной глубины резания.

Анотація. Розроблена динамічна модель пружної системи токарного верстата з врахуванням контактної взаємодіі заготовки з інструментом. Отримані аналітичні залежності для обчислення частот власних коливань пружної системи верстата при врахуванні процесу різання у вигляді додаткового пружного зв'язку в місиі контакту заготовки 3 інструментом. Наведена методика обчислення жорсткості иього зв'язку з урахуванням пружних зміщень заготовки $i$ інструменту. На токарно-гвинторізному верстаті мод. 16Б05П експериментально визначені частоти власних коливань шпинделя з закріпленою в патроні заготовкою без ї̈ підтисканням різцем і при п'яти варіантах підтискання на різних вигонах. Для иього ж верстата, і таких же умов взаємодї заготовки з різцем, проведено чисельне моделювання частот 
власних коливань пружної системи верстата. Результати моделювання відповідають експериментальним значенням частот власних коливань і достатньо точно відображають тендениї їх зміни при зміні масо-інериійних характеристик заготовки $і$ умов ї̈ взаємодії з різцем. Це підтверджує доцільність врахування процесу різання в замкнених динамічних моделях металорізальних верстатів у вигляді додаткового пружного зв'язку в місиі контакту заготовки і інструмента Ключові слова: токарний верстат; ипиндельний вузол; динамічна модель; частоти власних коливань; експеримент; чисельне моделювання.

Abstract. Dynamical model of lathe elastic system, including contact interaction of workpiece and tool, has been developed. Has been obtained analytical dependencies for natural-vibration frequencies calculation of the machine-tool elastic system, including cutting process in a form of additional elastic joint, positioned in the point of workpiece and tool contact. Design procedure of stiffness calculation including elastic displacements of workpiece and tool has been introduced. Natural-vibration frequencies for the spindle of engine lathe $16505 \Pi$ has been experimentally obtained for cases: workpiece fastened in the draw-in gear of the spindle unit without pressing it by tool; five cases of fastened workpiece pressed by tool on different overhanging lengths. For same lathe and interaction conditions of workpiece and tool, numerical simulation of natural-vibration frequencies of machine-tool elastic system has been performed. Results of simulation correspond with experimental values of natural-vibration frequencies and display precisely enough tendencies of their variation, due to workpiece mass and inertia characteristics variation and its interaction conditions with a tool. Obtained results validates appropriateness of this design procedure in the closed-loop dynamic models of metal-cutting tools, by taking into consideration cutting process as an additional elastic joint, positioned in the point of workpiece and tool contact.

Keywords: lathe; spindle unit; dynamic model; natural-vibration frequencies; experiment; numerical simulation.

\section{Библиографический список использованной литературы}

1. Lin C.-W. Dynamic models and design of spindle-bearing systems of machine tools: A review / C. W. Lin, Y.K. Lin, C. H. Chu // International Journal of Precision Engineering and Manufacturing, - Vol. 14(3), 2013 - pp. 513-521.

2. Кудинов В.А. Динамика станков / В.А. Кудинов - М.: Машиностроение, 1967.- 360 с.

3. Гусев В.В. Обоснование расчетной схемы для исследования динамического состояния технологической системь алмазно-абразивной обработки тонкостенных оболочек из ситалла / В.В. Гусев, Л.П. Калафатова, С.Ю. Олейник // Сучасні технології в машинобудуванні. - Харьков: Изд-во НТУ "ХПИ", 2011. - С. 14-23.

4. Иванов О.И. Численные и экспериментальные исследования динамических явлений прочесса механической обработки маложестких валов / О.И. Иванов, О.И. Драчев, Д.В. Гранченко // Вектор науки Тольяттинского государственного университета - № 2 (24), 2013. - С. 144-148.

5. Данильченко М.А. Учет процесса резания в динамических моделях шииндельных узлов металлорежущих станков / М.А. Данильченко // Надійність інструменту та оптимізація технологічних систем. Збірник наукових праць. Краматорськ. - 2016. - №38. - C. 99-104.

6. Abele E. Machine Tool Spindle Units / E. Abele, Y. Altintas, C. Brecher. // CIRP Annals-Manufacturing Technology - Vol. 59, No. 2, 2010. - pp.781-802.

7. Данильченко Ю.М. Исследование динамических характеристик механической системы «шпиндельный узел» / Ю.М. Данильченко, А.О. Дорожко, А.И. Петришин // Вестник МГТУ «Станкин» - № 1 (28), 2014. - С. 81-91.

8. Бидерман В.Л. Прикладная теория механических колебаний: Учеб. пособие для втузов / В.Л. Бидерман - М.: Машиностроение, 1972. - $416 \mathrm{c.}$

9. Ивович В.А. Переходные матриць в динамике упругих систем: Справочник. - М.: Машиностроение, 1981. - 183 с.

10. Петраков Ю.В. Моделирование прочессов резания: учебное пособие / Петраков Ю.В., Драчев О.И. - Старый Оскол: THT, 2011. $-240 \mathrm{c}$.

\section{References}

1. Lin, C.-W., Lin, Y.-K., and Chu, C.-H. (2013), Dynamic models and design of spindle-bearing systems of machine tools: A review, International Journal of Precision Engineering and Manufacturing, vol. 14 (March), pp. 513-521.

2. $\quad$ Kudinov, V.A. (1967), Dinamika stankov, Mashinostroenie, Moscow, Russian.

3. Gusev, V.V., Kalafatova, L.P. and Olejnik, S.Ju. (2011), Obosnovanie raschetnoj shemy dlja issledovanija dinamicheskogo sostojanija tehnologicheskoj sistemy almazno-abrazivnoj obrabotki tonkostennyh obolochek iz sitalla, Suchasni tehnologiï $v$ mashinobuduvanni, Izd-vo NTU "HPI", Har'kov, Ukraina, pp. 14-23.

4. Ivanov, O.I., Drachev, O.I. and Granchenko, D.V. (2013), Chislennye i jeksperimental'nye issledovanija dinamicheskih javlenij processa mehanicheskoj obrabotki malozhestkih valov, Vektor nauki Tol'jattinskogo gosudarstvennogo universiteta vol. 2 (24). pp. 144-148.

5. Danylchenko, M.A. (2016), Uchet processa rezanija v dinamicheskih modeljah shpindel'nyh uzlov metallorezhushhih stankov, Nadijnist' instrumentu ta optimizacija tehnologichnih sistem. Zbirnik naukovih prac', Kramators'k, Ukraina vol. 38 , pp. 99-104.

6. Abele, E., Altintas, Y. and Brecher, C. (2010), Machine Tool Spindle Units, CIRP Annals-Manufacturing Technology, vol. 59, No. 2, pp.781-802.

7. Danylchenko, Ju.M., Dorozhko, A.O. and Petryshyn, A.I. (2014), Issledovanie dinamicheskih harakteristik mehanicheskoj sistemy «shpindel'nyj uzel», Vestnik MGTU «Stankin», no 1 (28), pp. 81-91.

8. Biderman, V.L. (1972), Prikladnaja teorija mehanicheskih kolebanij: Ucheb. posobie dlja vtuzov. Mashinostroenie, Moscow, Russian.

9. Ivovich, V.A. (1981), Perehodnye matricy v dinamike uprugih sistem, Spravochnik, Mashinostroenie, Moscow, Russian.

10. Petrakov, Ju.V. and Drachev, O.I. (2011), Modelirovanie processov rezanija, uchebnoe posobie, Staryj Oskol, TNT. 